Relations industrielles

Industrial Relations

\title{
Gestion de l'entreprise, par Jane Aubert-Krier, Presses universitaires de France, Paris, 1962, 615 pages.
}

\section{Bertrand Belzile}

Volume 20, numéro 3, 1965

URI : https://id.erudit.org/iderudit/027594ar

DOI : https://doi.org/10.7202/027594ar

Aller au sommaire du numéro

Éditeur(s)

Département des relations industrielles de l'Université Laval

ISSN

0034-379X (imprimé)

1703-8138 (numérique)

Découvrir la revue

Citer ce compte rendu

Belzile, B. (1965). Compte rendu de [Gestion de l'entreprise, par Jane Aubert-Krier, Presses universitaires de France, Paris, 1962, 615 pages.] Relations industrielles / Industrial Relations, 20(3), 575-576.

https://doi.org/10.7202/027594ar

Tous droits réservés @ Département des relations industrielles de l'Université Laval, 1965
Ce document est protégé par la loi sur le droit d'auteur. L’utilisation des services d'Érudit (y compris la reproduction) est assujettie à sa politique d'utilisation que vous pouvez consulter en ligne.

https://apropos.erudit.org/fr/usagers/politique-dutilisation/ 


\section{RECENSIONS BOOKS REVIEW}

Manogement: Challenge and Response, par Mortin B. Corter et ol. (Eds), Holt, Rinehart and Winston, Inc., Toronto, 1965, 274 poges.

Depuis un bon nombre d'années la revue Fortune publie une brève histoire des grandes entreprises privées américaines, ainsi qu'un profil des chefs qui en ont ossuré la survie ou milieu des épreuves et des crises qu'elles ont offrontées.

Dans ce volume, les éditeurs ont rassemblé probablement les meilleurs articles de la série, afin de permettre ò l'étudiant en administration des entreprises d'établir un pont entre la théorie et la pratique. De fait, une étude personnelle des cas permet de mieux saisir comment des odministrateurs chevronnés ont dirigé et dirigent encore les activités différenciées qui se déploient ou sein de la grande organisation. Une analyse intensive et comparative de chaque cas permet aussi de saisir la façon dont les décisions sont prises à un moment critique et la personnalité de ceux qui les prennent.

\section{Laurent Bélanger}

Regulating Union Government, Marten S. Estey, Philip Taft, Martin Wagner, Editors, Harper \& Row Publishers, New York, Evanston and London, 1964, 230 pages.

Ce volume est le fruit de la collaboration de Joel Seidman, Sar A. Leviton, J. Joseph Loewenberg, Benson Soffer, Emanuel Stein, Howard Jenkins, Jr. and D. Stewart. Chacun de ces auteurs a écrit un chapitre oyant trait de foçon directe ou indirecte au - Labor-Management Reporting and Disclosure Act de 1959, mieux connu sous le nom de Loi Landrum-Griffin. Les éditeurs ont tenté de grouper une série de chapitre pour foire ressortir l'arrière-plan de cette loi, la façon dont elle est administrée, ce qu'elle a permis d'apprendre sur la conduite et l'administration des syndicats, et ses conséquences sur lo convention collective.

Dans un premier chapitre, Seidman étudie les critiques croissantes qui 's'élevaient contre la corruption syndicale ovant l'adoption du LMRDA. De leur côté, Leviton et Loewen- berg donnent une description des enquêtes sénatoriales et des problèmes juridiques qui ont précédé la loi. Dans un troisième chapitre, Stewart donne une description et une appréciation de l'organisation, des fonctions et des activités du « Bureau of Labor-Manogement Reports . Soffer, lui, conclut que la loi n'a eu que des effets négligeables sur la convention collective et il prévoit qu'il en sera de même dans les années ò venir. Les implications du LMRDA sur les finances des syndicats sont étudiées par Stein. Dans le chapitre suivant, Jenkins démontre que la loi n'est encore qu'une tentative de démocratisation $\triangleright$, en ce qui concerne les élections dans les unions de métier. En dernier lieu, Marten S. Estey commente les recherches et les stotistiques du BLMR durant les quatre premières années de son entrée en vigueur.

D'une lecture facile à couse de son style bien approprié, cet ourrage constitue une excellente critique des évènements syndicaux et juridiques qui ont conduit à l'adoption de la loi Landrum-Griffin en 1959, des répercussions et de l'efficacité de cette loi sur diverses activités syndicoles, en particulier les finances, les élections et la convention collective.

Cependant, les outeurs le reconnaissent aussi, il est trop tôt pour déterminer les effets précis de la loi sur la conduite des syndicats. Mais les recherches de ces auteurs permettent déjà de déceler les faiblesses et l'efficacité de la loi, ainsi que de vérifier si les accusations portées contre les syndicats ovant 1959 étaient fondées ou non. Malheureusement, il semble que le BLMR ne fournit pas beaucoup de données sur les structures syndicales et les effectifs oux différents niveaux de ces structures.

\section{Marc Poulin}

Gestion de I'entreprise, por Jane AubertKrier, Presses universitaires de France, Paris, 1962, 615 poges.

L'auteur affirme dans son introduction que celui qui l'aborde (l'enseignement de l'administration) doit éviter trois écueils: tout traiter mais de foçon trop sommaire, aborder les difficultés mais de façon telle- 
ment ollusive que ce soit incompréhensible, ne traiter qu'une portie du progromme Est-il possible d'éviter les trois écueils? Nous ne le croyons pas. En effet, la gestion de l'entreprise o comme objet la vie même de l'entreprise, dans ses activités concrètes, multiples et variées. A la rigueur, il est humoinement, ou du moins pratiquement impossible, de cerner tous les aspects reliés aux octivités de l'entreprise.

On répète souvent à juste titre que lo gestion de lo firme est une science-carrefour. L'outeur de son propre dire, n'a donc pu éviter, pos plus que les outres d'ailleurs, les trois écueils mentionnés plus hout. II a * choisi délibérément de développer dovantage certaines porties, comme la structure et l'orgonisation des entreprises $\gg$ qui foit l'objet de la deuxième partie. On y retrouve d'abord un bon exposé des doctrines de Taylor, Fayol et Ford, puis des fonctions de l'entreprise et des services qui les remplissent. Enfin, les deux outres chopitres de cette portie traitent très abondamment de lo structure et de l'organisation interne.

Lo première portie de l'ouvroge couvre les formes et classification des entreprises. C'est à coup sûr celle qui opportera le moins à lo pluport des lecteurs.

Lo troisième portie touche un aspect primordial d'une soine gestion, l'information. Celle-ci constitue probablement l'obstocle principol à lo prise de décisions vroiment éclairée. Et pourtont, prise de décision n'est-il pas le mot clef de l'odministration! Les sujets que l'outeur courre sous cette rubrique nous opporoissent un bon choix; ce sont la méthode budgétaire et le tableau de bord.

L'activité de l'entreprise forme la dernière portie qui traite du produit, de la vente et des moyens (les problemes financiers et les hommes) en trois chapitres différents. A peu près tous les sujets $y$ sont couverts, mais forcément d'une foçon assez superficielle.

Cette dernière remorque coractérise assez bien l'ensemble du volume qui a une ollure plutôt encyclopédique. Toutefois, ceci rend l'ouvrage de modame Aubert-Krier très accessible aux non-initiés d la science administrative. Et de ce foit, «estion de l'entreprise vaut bien d'autres livres sur le même sujet

\section{Bertrond Belzile}

Decision Making in the Employment Interview, par Edward C. Webster, McGill University, Industrial Relotions Centre, Montreal, 1964, 124 pages.
Un condidot qui se voit refuser une position peut douter de lo qualité de lo décision formulée à son égord. Une lecture ropide du volume du Professeur Webster ne peut que confirmer cet énoncé, puisqu'il jette un éclairage nouveau sur la foçon dont un préposé à la sélection du personnel arrive à une decision touchant l'occeptotion ou le refus d'un condidat. L'auteur ropporte d'une focon concise les résultats de cinq thèses doctoroles présentées ò l'université et foit ressortir les conclusions mojeures de chaque projet de recherche.

Un même condidat peut être accepté ou refusé par différents interviewers, tout dépend d'un ensemble de focteurs qui influencent celui qui doit prendre la décision. L'interviewer possède au préalable, une notion de ce qu'est un «bon candidat

Si cette notion se crystallise ou point de ne plus correspondre oux exigences modifiées des tôches, lo décision d'accepter ou de refuser un condidat sero offectée.

Lo première impression que se fait l'interviewer va colorer oussi so décision, puisqu'il tend à centrer son ottention sur l'information qui peut la confirmer. De plus l'interviewer tend à donner plus de poids à des coroctéristiques qui défavorisent le candidat. La distance sociole entre l'interviewer et le candidat, l'ordre de la présentation de l'information constituent d'autres variables qui conditionnent la décision.

L'auteur situe les résultats de ces travaux dans le contexte plus vaste de la recherche portant sur les focteurs physiques et psychologiques qui influencent la perception.

En conclusion, quelques conseils pratiques sur la façon d'améliorer la prise de décision dans le domaine de la sélection du personnel méritent d'être retenus.

\section{Laurent Bélanger}

Principles of Economics, Second Edition, G.E. Ferguson \& I.M. Kreps, Holt, Rinehart and Winston Inc., Toronto, 1965, 863 poges

- I hove called for a notional war on poverty. " (President Kennedy)

Cette phrose réflète le but de tous les poys du monde pour les années soixante: le développement économique. Ainsi, dons notre économie d'abondance, il n'en reste pos moins que plusieurs vivent dans la pourreté et la misère. Est-ce un paradoxe? 